\title{
TRIALS
}

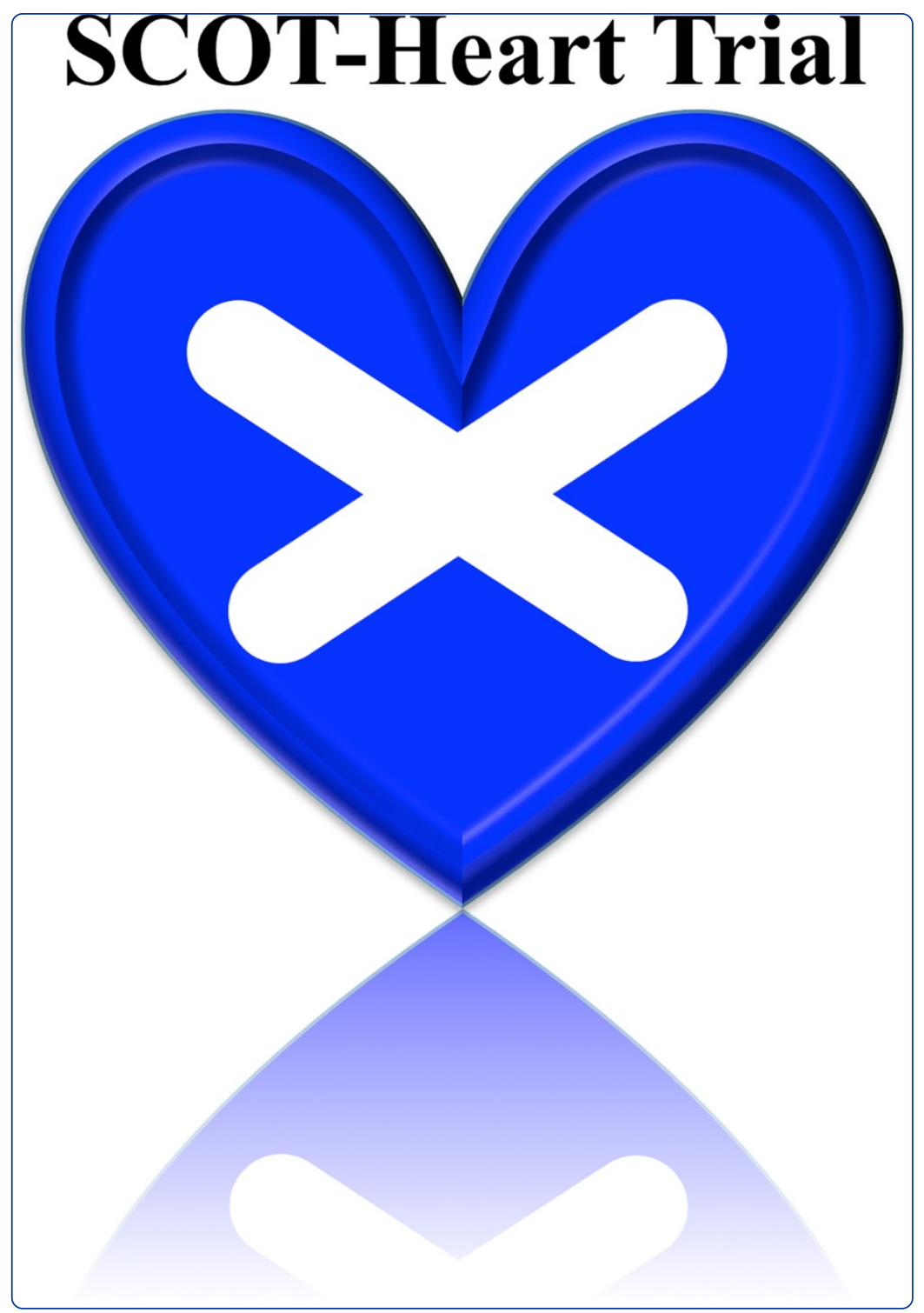

Role of multidetector computed tomography in the diagnosis and management of patients attending the rapid access chest pain clinic, The Scottish computed tomography of the heart (SCOT-HEART) trial: study protocol for randomized controlled trial Newby et al. 


\section{Role of multidetector computed tomography in the diagnosis and management of patients attending the rapid access chest pain clinic, The Scottish computed tomography of the heart (SCOT-HEART) trial: study protocol for randomized controlled trial}

David E Newby ${ }^{1}$, Michelle C Williams ${ }^{1 *}$, Andrew D Flapan², John F Forbes ${ }^{3}$, Allister D Hargreaves ${ }^{4}$, Stephen J Leslie ${ }^{5}$, Steff C Lewis ${ }^{6}$, Graham McKillop ${ }^{2}$, Scott McLean ${ }^{7}$, John H Reid ${ }^{8}$, James C Sprat ${ }^{4}$, Neal G Uren², Edwin J van Beek ${ }^{9}$, Nicholas A Boon ${ }^{1}$, Liz Clark ${ }^{10}$, Peter Craig ${ }^{11}$, Marcus D Flather ${ }^{12}$, Chiara McCormack ${ }^{6}$, Giles Roditi ${ }^{13,14}$, Adam D Timmis ${ }^{15}$, Ashma Krishan ${ }^{6}$, Gillian Donaldson ${ }^{8}$, Marlene Fotheringham ${ }^{8}$, Fiona J Hall ${ }^{8}$, Paul Neary ${ }^{8}$, Louisa Cram ${ }^{4}$, Sarah Perkins ${ }^{4}$, Fiona Taylor ${ }^{4}$, Hany Eteiba ${ }^{13}$, Alan P Rae ${ }^{13}$, Kate Robb ${ }^{13}$, Dawn Barrie ${ }^{16}$, Kim Bissett ${ }^{16}$, Adelle Dawson ${ }^{16}$, Scot Dundas ${ }^{16}$, Yvonne Fogarty $^{16}$, Prasad Guntur Ramkumar ${ }^{16}$, Graeme J Houston ${ }^{16}$, Deborah Letham ${ }^{16}$, Linda O'Neill ${ }^{16}$, Stuart D Pringle ${ }^{16}$, Valerie Ritchie ${ }^{16}$, Thiru Sudarshann ${ }^{16}$, Jonathan Weir-McCall ${ }^{16}$, Alistair Cormack ${ }^{17}$, lain N Findlay ${ }^{17}$, Stuart Hood ${ }^{17}$, Clare Murphy ${ }^{17}$, Eileen Peat ${ }^{17}$, Barbara Allen ${ }^{9}$, Andrew Baird ${ }^{9}$, Danielle Bertram9, David Brian9, Amy Cowan ${ }^{9}$, Nicholas L Cruden², Marc R Dweck', Laura Flint ${ }^{2}$, Samantha Fyfe ${ }^{9}$, Collette Keanie ${ }^{2}$, Tom J MacGillivray ${ }^{9}$, David S Maclachlan', Margaret MacLeod ${ }^{2}$, Saeed Mirsadraee ${ }^{9}$, Avril Morrison 2,18, Nicholas L Mills ${ }^{1}$, Fiona C Minns ${ }^{2}$, Alyson Phillips ${ }^{2}$, Laura J Queripel ${ }^{9}$, Nicholas W Weir ${ }^{2,9}$, Fiona Bett ${ }^{19}$, Frances Divers ${ }^{19}$, Katie Fairley ${ }^{19}$, Ashok J Jacob ${ }^{19}$, Edith Keegann ${ }^{19}$, Tricia White ${ }^{19}$, John Gemmill ${ }^{20}$, Margo Henry ${ }^{20}$, James McGowan ${ }^{20}$, Lorraine Dinnel $^{21}$, C Mark Francis ${ }^{21}$, Dennis Sandeman ${ }^{21}$, Ajay Yerramasu ${ }^{21}$, Colin Berry ${ }^{14}$, Heather Boylan ${ }^{14}$, Ammani Brown ${ }^{14}$, Karen Duffy ${ }^{14}$, Alison Frood ${ }^{14}$, Janet Johnstone ${ }^{14}$, Kirsten Lanaghan ${ }^{14}$, Ross MacDuff ${ }^{14}$, Martin MacLeod ${ }^{14}$, Deborah McGlynn ${ }^{14}$, Nigel McMillan ${ }^{14}$, Laura Murdoch ${ }^{14}$, Colin Noble ${ }^{14}$, Victoria Paterson ${ }^{14}$, Tracey Steedman ${ }^{14}$ and Nikolaos Tzemos ${ }^{14}$

\section{Abstract}

Background: Rapid access chest pain clinics have facilitated the early diagnosis and treatment of patients with coronary heart disease and angina. Despite this important service provision, coronary heart disease continues to be under-diagnosed and many patients are left untreated and at risk. Recent advances in imaging technology have now led to the widespread use of noninvasive computed tomography, which can be used to measure coronary artery calcium scores and perform coronary angiography in one examination. However, this technology has not been robustly evaluated in its application to the clinic.

(Continued on next page)

\footnotetext{
*Correspondence: michelle.williams@ed.ac.uk

'University of Edinburgh/BHF Centre for Cardiovascular Science, Chancellor's

Building, 49 Little France Crescent, Edinburgh EH16 SU4, UK

Full list of author information is available at the end of the article
} 
(Continued from previous page)

Methods/design: The SCOT-HEART study is an open parallel group prospective multicentre randomized controlled trial of 4,138 patients attending the rapid access chest pain clinic for evaluation of suspected cardiac chest pain. Following clinical consultation, participants will be approached and randomized 1:1 to receive standard care or standard care plus $\geq 64$-multidetector computed tomography coronary angiography and coronary calcium score. Randomization will be conducted using a web-based system to ensure allocation concealment and will incorporate minimization. The primary endpoint of the study will be the proportion of patients diagnosed with angina pectoris secondary to coronary heart disease at 6 weeks. Secondary endpoints will include the assessment of subsequent symptoms, diagnosis, investigation and treatment. In addition, long-term health outcomes, safety endpoints, such as radiation dose, and health economic endpoints will be assessed. Assuming a clinic rate of $27.0 \%$ for the diagnosis of angina pectoris due to coronary heart disease, we will need to recruit 2,069 patients per group to detect an absolute increase of $4.0 \%$ in the rate of diagnosis at $80 \%$ power and a two-sided $P$ value of 0.05 . The SCOT-HEART study is currently recruiting participants and expects to report in 2014.

Discussion: This is the first study to look at the implementation of computed tomography in the patient care pathway that is outcome focused. This study will have major implications for the management of patients with cardiovascular disease.

Trial registration: ClinicalTrials.gov Identifier: NCT01149590

Keywords: Computed tomography, Coronary heart disease, Rapid access chest pain clinic

\section{Background}

The clinical presentation of chest pain is a major problem for primary health care professionals and is the commonest medical reason for a patient attending the Emergency Department. Ascertaining the aetiology of the chest pain is essential not only for the future management and investigation of the patient, but also for health care resources to be utilized appropriately and efficiently. The distinction between cardiac and noncardiac chest pain can be subtle, leading, in some series $[1,2]$, to between 2 and $12 \%$ of patients being inappropriately discharged from hospital and more than $25 \%$ being readmitted to hospital with benign noncardiac chest pain. From the primary care perspective, Emergency Department attendances or short-term hospitalizations with an unhelpful diagnosis, such as 'chest pain - myocardial infarction excluded', do not provide a clear diagnosis or management plan.

\section{Rapid access chest pain clinics}

The accurate identification of patients with ischaemic heart disease is important because up to $30 \%$ of patients presenting with recent-onset angina have a cardiac event within 1 to 2 years [3] and many of these patients may benefit from coronary revascularization [4]. This has led many centres to develop the provision of a rapid access chest pain clinic. This out-patient clinic provides a 'onestop' assessment for patients with suspected angina, including medical history, examination, electrocardiogram, blood tests, and exercise testing where appropriate. It does not include patients with acute chest pain who require immediate hospital assessment for suspected acute coronary syndrome. We have demonstrated that such services reduce the hospitalization of patients with benign noncardiac chest pain whilst facilitating the identification of those patients with acute coronary syndromes requiring in-patient care [5]. A specialist cardiology opinion combined with the resources of a chest pain clinic service would appear to have a higher diagnostic yield for ischaemic heart disease than open-access exercise electrocardiography, and would provide the primary care physician with a firm clinical diagnosis in the majority of cases, and identify those patients requiring further invasive investigation [5].

\section{The need for better diagnostic accuracy and risk stratification}

Rapid access chest pain clinics have now become established across the United Kingdom and they have proven successful in identifying high-risk patients with coronary heart disease [6]. However, there is room for improvement, with some patients continuing to be misdiagnosed with noncardiac chest pain [6]. Moreover, those diagnosed with noncardiac chest pain account for up to a third of patients who subsequently die from cardiovascular disease or suffer an acute coronary syndrome over 5 years of follow-up [6]. There is, therefore, a need for better diagnostic accuracy and risk stratification in patients attending rapid access chest pain clinics, especially in younger patients $(<65$ years) [7].

\section{Coronary artery calcification}

Coronary artery calcification is an independent risk factor for coronary heart disease, with even low coronary 
calcium scores doubling the risk of coronary events [8]. The relative risk associated with coronary calcification is greater than that associated with established factors, such as smoking, hypertension and diabetes mellitus. The progression of coronary artery calcification is associated with a higher incidence of coronary events, even in those people who are asymptomatic at the time of initial scanning [9]. Thus, the presence of coronary artery calcification is not only indicative of atheromatous plaque disease, but its progression may correspond with cardiovascular event rates.

The degree of calcification correlates with atherosclerotic burden but it does not identify soft plaque and may not predict the patient's response to medical interventions $[10,11]$. Moreover, the presence of coronary artery calcification does not, in itself, predict the presence of obstructive atheroma. Calcification can, therefore, be used as a surrogate marker of the extent of coronary atherosclerotic disease, rather than as a measure of luminal stenosis [12].

\section{Computed tomography coronary angiography}

Major advances in scanning technology have led to the establishment of noninvasive coronary angiography by multidetector computed tomography (MDCT). This has a very good agreement with invasive coronary angiography $[13,14]$ and intravascular ultrasound [13-16], with kappa coefficient values of 0.75 for both. The resolution of modern scanners allows quantification of luminal stenoses as well as identification of noncalcified 'soft' atherosclerotic plaque [15]. Pooled analysis of over 800 patients indicates a sensitivity of $89 \%$ ( $95 \%$ confidence intervals, 87 to $90 \%$ ) and specificity of $96 \%$ ( $95 \%$ confidence intervals, 96 to 97\%) for 64-MDCT in comparison with invasive coronary angiography [16]. The major strength is in the negative predictive value of $98 \%$ (95\% confidence intervals, 98 to 99\%). The current evolution of scanning technology has led to greater spatial and temporal resolution with lower radiation doses ( $\sim 2$ to $3 \mathrm{mSv}$ ). This should translate into a highly effective and safe imaging strategy, particularly for the evaluation of stable patients with possible coronary artery disease [17].

It is important to highlight that computed tomography (CT) coronary angiography is primarily used to confirm or refute the presence of coronary artery disease. The diagnosis of angina pectoris due to coronary heart disease primarily relies on two factors: (i) a history consistent with angina pectoris, and (ii) the presence of obstructive coronary artery disease. It should be realized that patients with coronary heart disease may have nonanginal chest pain and that patients with typical anginal chest pain may not have coronary heart disease (see Table 1).

A health technology assessment including a comprehensive systematic review of 64-multidetector CT coronary angiography highlighted several areas that require further research, including (i) the usefulness of MDCT coronary angiography in patients with suspected coronary artery disease; (ii) the advantages of 256versus 64-MDCT coronary angiography; and (iii) the role of MDCT to assess coronary artery plaque morphology [18]. In addition, the National Institute of Clinical Excellence (NICE) specifically called for research into the clinical efficacy and cost-effectiveness of MDCT coronary angiography compared with functional testing in the diagnosis of angina [19].

\section{Methods/design Study design}

This is an open parallel group prospective randomized controlled trial, assessing the impact of CT on the diagnosis and management of patients attending a rapid access chest pain clinic.

\section{Study objectives}

The purpose of a rapid access chest pain clinic is to identify patients with symptoms of angina attributable to coronary heart disease, in order to identify those who would benefit from secondary prevention and antianginal therapies. The standard approach is to document a clinical history of angina pectoris and demonstrate objective evidence of exercise-induced myocardial ischaemia through exercise stress testing. We wish to evaluate the added value of coronary artery calcium scoring and $\mathrm{CT}$ coronary angiography in the assessment of patients attending a rapid access chest pain clinic.

In the setting of a rapid access chest pain clinic, the most important question that the patient has is whether his or her chest pain is due to coronary heart disease. The primary objective of the study, therefore, is to investigate whether the inclusion of coronary artery calcium scoring and CT coronary angiography alters the proportion of patients diagnosed with angina due to coronary heart disease at 6 weeks.

The secondary objectives of the study are to ascertain whether a coronary artery calcium score and CT coronary angiogram influences the management of patients with coronary heart disease or noncardiac causes of chest pain. Patients' concerns often relate to the chest pain itself (symptoms), what causes their symptoms (diagnosis), what further tests are required (investigations), what medication or procedures are recommended (treatments) and what the impact will be on their future health (long-term outcomes). We will also assess safety and health economic outcomes. We will undertake longterm follow of these patients to determine whether CT assessments independently predict future risk, and whether this leads to improved clinical outcomes through better guided use of therapies. 
Table 1 Categorization of patients based on presence the presence or absence of angina pectoris and coronary heart disease

\begin{tabular}{llll}
\hline & Coronary heart disease & \\
\cline { 2 - 3 } & & Yes & No \\
\hline Angina pectoris & Yes & High-risk and cause of symptoms & Low-risk and cause of symptoms unclear \\
\cline { 2 - 3 } & No & High-risk but either symptoms unrelated or atypical presentation & Low-risk and other cause of symptoms likely \\
\hline
\end{tabular}

\section{Primary endpoint}

The primary endpoint of the study will be the proportion of patients diagnosed with angina pectoris secondary to coronary heart disease at 6 weeks. The clinician in charge of the patients' care will assign the diagnosis following either (a) analysis of the coronary artery calcium score and CT coronary angiogram (intervention group), or (b) standard care (conservative group).

\section{Secondary endpoints}

The following secondary endpoints will be evaluated: (i) the frequency and severity of chest pain symptoms at six weeks and six months; (ii) the CT observed presence and extent of coronary artery disease; (iii) the diagnosis and severity of coronary heart disease; (iv) the accuracy of CT coronary angiography compared with the goldstandard of invasive coronary angiography in those who receive both investigations; (v) the effect on investigations, unscheduled care and healthcare resource utilization; and (vi) the effect on patient management, including secondary prevention, anti-anginal therapy (pharmacological therapy and coronary revascularization), and treatment of noncardiac chest pain (such as hiatus hernia).

The study has been primarily set up to assess a patient-focused symptom outcome. However, alterations in management may result in long-term benefits to patients. Therefore long-term outcomes will be assessed, including: (i) cardiovascular death or nonfatal myocardial infarction; (ii) cardiovascular death; (iii) nonfatal myocardial infarction (universal definition); (iv) cardiovascular death, nonfatal myocardial infarction or nonfatal stroke; (v) nonfatal stroke; (vi) all causes of death; (vii) coronary revascularization, percutaneous coronary intervention or coronary artery bypass graft surgery; (viii) hospitalization for chest pain, including acute coronary syndromes and noncoronary chest pain; and (ix) hospitalization for cardiovascular disease, including coronary artery disease, cerebrovascular disease and peripheral arterial disease.

\section{Patient population}

Participants will be identified from patients attending the rapid access chest pain clinic. We will recruit 4,138 patients, randomized $1: 1$ to standard care $(n=2,069)$ or standard care with coronary calcium score and CT coronary angiography $(n=2,069)$.

Inclusion criteria will be: (i) attendance at the rapid access chest pain clinic and (ii) age over 18 years but less than or equal to 75 years.

Exclusion criteria will be: (i) inability or unwillingness to undergo CT scanning; (ii) exceeding the weight tolerance of scanner; (iii) known severe renal failure (serum creatinine $>200 \mu \mathrm{mol} / \mathrm{l}$ or estimated glomerular filtration rate $<30 \mathrm{ml} / \mathrm{min}$ ); (iv) previous recruitment to the trial; (v) major allergy to iodinated contrast agent; (vi) inability to give informed consent; (vii) known pregnancy; or (viii) acute coronary syndrome within 3 months.

\section{Participant selection and enrolment}

The trial is a pragmatic evaluation of the added value of CT coronary angiography in a rapid access chest pain clinic. Only patients attending the rapid access chest pain clinic will be approached to participate. The inclusion and exclusion criteria are broad and inclusive, and should enable rapid identification of eligible patients attending the clinic.

Patients will be given a patient information sheet on arrival at the rapid access chest pain clinic. After consultation, all eligible patients will be approached to enter the trial by the attending clinician. Written informed consent will be obtained from patients willing to participate in the study (Figure 1).

\section{Randomization}

Following recruitment, patients who agree to undergo research evaluation will be randomized (1:1), either to no additional scanning or to undergo further evaluation with coronary calcium scoring and CT coronary angiography within 14 days of clinic attendance and before invasive coronary angiography or other cardiac investigations. Randomization will be conducted using a web-based system to ensure allocation concealment and will incorporate minimization to ensure matching for age, sex, body mass index (height and weight), diabetes mellitus, prior history of coronary heart disease, atrial fibrillation and baseline diagnosis of angina due to coronary heart disease.

\section{Equipoise}

Attending clinicians in the rapid access chest pain clinic will not be permitted to investigate patients with CT 


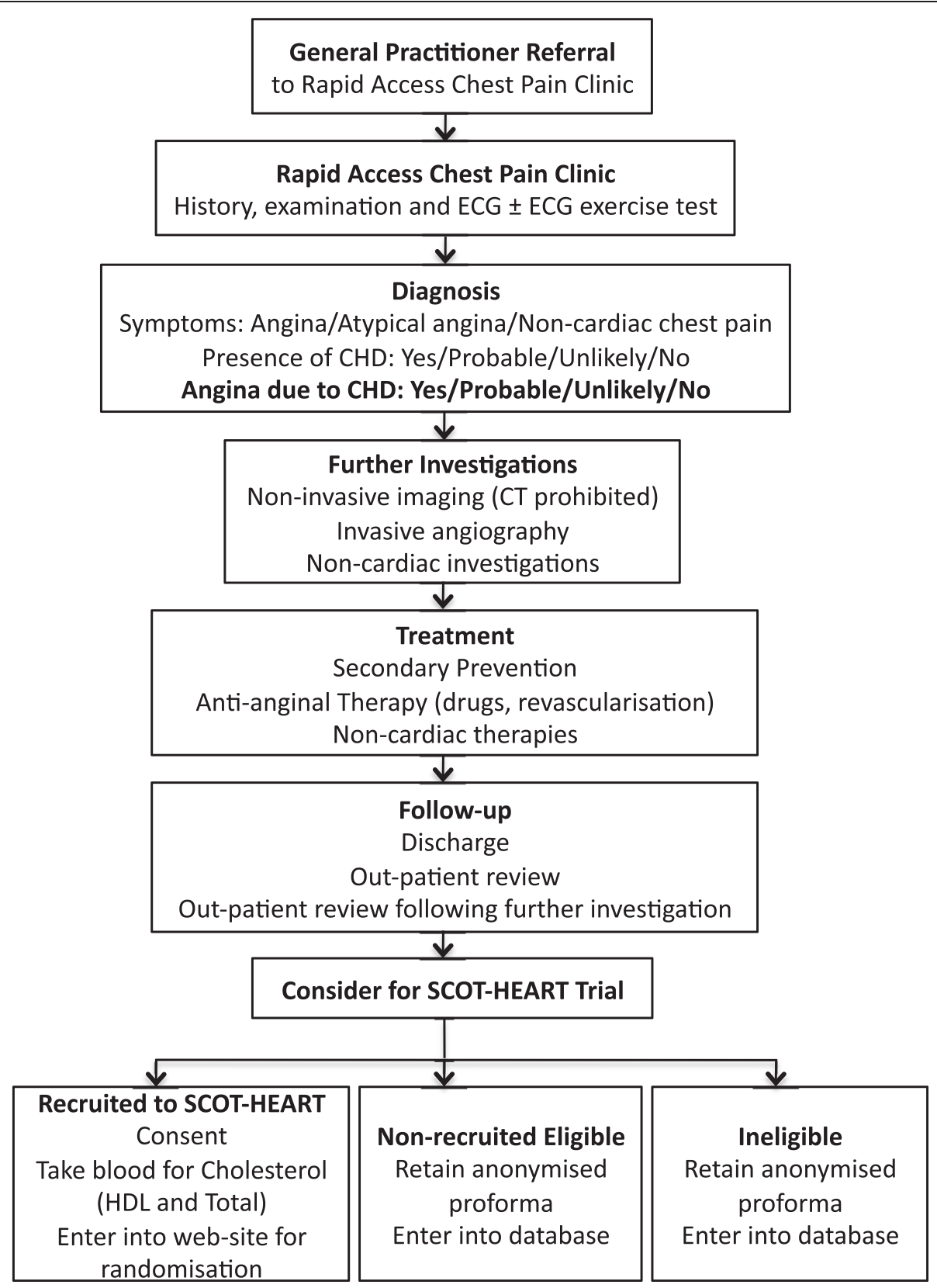

Figure 1 Rapid access chest pain clinic attendance, eligibility and recruitment. (CHD, coronary heart disease; CT, computed tomography; ECG, electrocardiogram; HDL, high dependency lipoprotein).

coronary angiography unless the patient is randomized to receive the scan as part of the trial. During the course of the trial, it is anticipated that CT coronary angiography may start to be introduced into routine clinical practice within the rapid access chest pain clinic setting. It will be important that loss of equipoise does not prejudice the trial. This practice will, therefore, be discouraged during the course of the trial. Where this is not feasible, either the trial centre will be closed or all patients will be approached to participate in the trial but, where a patient is randomized to standard of care, they will be excluded from undergoing a CT coronary angiogram and an alternative noninvasive test will be selected. Equipoise will be monitored through data collected on unrecruited patients.

\section{Study assessments and data collection}

Clinic assessment

All patients will undergo routine evaluation at the rapid access chest pain clinic including, where appropriate, symptom-limited exercise electrocardiography using the standard Bruce protocol. Patients will be categorized as 
low, intermediate or high risk by the attendant clinician, informed by the NICE guideline [20]. In general, highrisk patients will be treated for coronary heart disease and undergo invasive coronary angiography (10\%), and low-risk patients (10\%) will be reassured and discharged. Intermediate-risk patients $(80 \%)$ will be treated and further investigated at the discretion of the clinician. In all cases, the diagnosis and agreed management strategy will be documented at the end of the clinic attendance. Cardiovascular risk will be calculated using previously established risk scores, such as the ASSIGN and Framingham scores.

The study data sheet will be used in the rapid access chest pain clinic to document patient history, examination and management plan for all patients (recruited and unrecruited). This will be completed by the attending clinician or nurse. This will also include assessment of eligibility. The study pro forma for all ineligible participants and eligible unrecruited participants will be retained in an anonymized form to provide detailed data on these patients in comparison with the study participant population. All study pro formas (recruited and unrecruited eligible and ineligible patients) and consent forms (recruited participants) will be collated by the trial manager and entered into the database.

\section{Blood tests}

If the participant has not had total cholesterol and HDL (high-density lipoprotein) cholesterol levels measured within the past three months, a blood test will be taken at the rapid access chest pain clinic. If the total cholesterol is above $7.0 \mathrm{mmol} / \mathrm{l}$ or the HDL is below 0.5 $\mathrm{mmol} / \mathrm{l}$, the primary care physician will be informed by letter, as this may warrant treatment irrespective of the patient's 10-year cardiovascular risk.

In a sub-group of patients, a venous blood sample will be obtained and stored for future assessment of biomarkers.

\section{Computed tomography}

Computed tomography scans will be performed using a 64, 128 or 320-multidetector scanner. Computed tomography protocol optimizations will be performed at all sites throughout the study, to optimize scanning parameters, such as radiation dose and contrast administration.

Medication Before calcium scoring, patients with a heart rate of greater than 60 beats $/ \mathrm{min}$ and systolic blood pressure $>110 \mathrm{mmHg}$ will receive rate-limiting medication. If a participant's heart rate is above 100 beats per minute despite rate-limiting medication, CT coronary angiography will not be performed. A small dose of oral diazepam may be prescribed for anxious patients, to improve heart rate control. Sublingual glyceryl trinitrate will be administered immediately prior to CT imaging.

Coronary artery calcium score Coronary calcium scoring will be performed prior to coronary angiography. Investigators blind to patient characteristics will conduct off-line analyses using automated computerized software programs that employ the Agatston scoring method [21] using a threshold of 130 Hounsfield units [11]. The calcium score percentile based on age and sex will be calculated using coronary artery calcium score distributions from the Multi-Ethnic Study of Atherosclerosis (MESA) [22]. This will be performed using a web-based calculator, available at http://www.mesa-nhlbi.org/Calcium/input. aspx. For patients younger than 45, 45 years will be used for the calculation of the calcium score percentile.

Computed tomography coronary angiography Coronary angiography will be conducted during contrast enhancement using pre-specified protocols (as recommended by the scanner manufacturers) during a single breath hold with prospective electrocardiographic gating as appropriate.

\section{Image assessment}

All CT and invasive coronary angiograms will be assessed by at least two trained observers. Angiograms (CT and invasive) will be reviewed independently and without prior knowledge of the alternate angiogram. Where there is disagreement between paired observers (but not modalities), angiograms will be reviewed and classified by consensus. Angiograms will be reported using the 15-segment model [23]. Significant stenosis due to coronary artery disease will be defined as a stenosis greater than $70 \%$ in one or more major epicardial vessels or greater than $50 \%$ in the left main stem [20]. Luminal cross-sectional area stenoses will be classified as normal $(<10 \%)$, haemodynamically insignificant (10$49 \%)$, intermediate $(50-70 \%)$, significant (greater than or equal to $70 \%)$ or total or subtotal occlusion (100\%).

\section{Definition of coronary artery disease}

Coronary artery disease will be classified as: (a) obstructive coronary artery disease, atherosclerotic plaque encompassing a luminal cross-sectional area of $\geq 70 \%$ in at least one major epicardial vessel; (b) nonobstructive coronary artery disease, either atherosclerotic plaque encompassing a luminal cross-sectional area of $<70 \%$ but $>10 \%$ in at least one major epicardial vessel, or a calcium score $>400$ AU (Agatston units) or $>90$ th percentile for age and sex; or (c) minimal or no coronary artery disease. Significant plaque burden is defined as atherosclerotic plaque causing $>10 \%$ luminal cross-sectional area stenosis. 


\section{Management recommendations}

Computed tomography scans will be reported locally by two trained observers (radiologist and cardiologist). The CT report will also include recommendations for management, including primary or secondary prevention. Treatment of angina due to coronary heart disease will be at the discretion of the responsible consultant. (Figures 2 and 3). For participants in the no-CT-scan group, the responsible consultant will be sent a letter detailing the patient's ASSIGN score.

\section{Symptom outcomes}

At baseline, 6 weeks and 6 months, chest pain will be assessed by the UK version of the Seattle Angina Questionnaire (SAQ-UK) [24] and quality of life will be assessed by the 12 -Item Short Form Health Survey $\left(\mathrm{SF}-12^{\circledR}\right)$. The standard (4-week) recall second version of the SF-12 ${ }^{\circledR}$ will be used (SF-12v2 $\left.{ }^{\mathrm{Tu}}\right)$. The SF-12 Physical Component Summary (PCS) and Mental Component Summary (MCS) scores will be calculated, along with utility scores based on the SF-6D algorithm.

Baseline questionnaires will be handed to the patient at the end of the rapid access chest pain clinic consultation. At 6 weeks and 6 months, questionnaires will be posted to participants, with telephone follow-up for nonresponders after two mailings two weeks apart.

\section{Diagnostic outcomes}

The proportion of patients diagnosed with coronary heart disease will be documented at baseline, after CT (where appropriate), after 6 weeks and after 6 months. This will be defined as (i) prior history of coronary heart disease (previous documented acute myocardial infarction (universal definition), obstructive coronary heart disease ( $\geq 70 \%$ luminal stenosis in at least one major epicardial vessel on invasive coronary angiography) or previous coronary revascularization (percutaneous coronary intervention or coronary artery bypass surgery)), (ii) clinical diagnosis of angina pectoris due to coronary heart disease, or (iii) obstructive or nonobstructive CT diagnosis of coronary heart disease. The extent of coronary heart disease will be determined by the number of vessels affected (none, one, two or three vessels diseased $(\geq 70 \%$ luminal stenosis of a major epicardial vessel)) and plaque load determined by CT coronary angiography.

The accuracy of the CT coronary angiography will be determined by comparison with invasive coronary angiography (gold-standard) for the assessment of the number of vessels affected. The extent of plaque burden cannot be compared between the two modalities.

\section{Investigation outcomes}

Relevant investigations will be documented for each participant at the baseline clinic attendance, after 6 weeks and after 6 months. This will include: an exercise electrocardiographic stress test; nuclear medicine imaging myocardial perfusion imaging; stress echocardiography; invasive coronary angiography; and noncardiac investigations, for example, endoscopy.

This will be documented by the clinician in charge of patient care in response to the CT coronary angiogram report. Further information will be obtained from electronic hospital records and patient surveys. Computed tomography coronary angiography will be performed prior to any invasive coronary angiogram, to facilitate a decision to undertake or cancel this invasive investigation.

\section{Treatment outcomes}

All treatments will be documented at baseline, after CT scan (where appropriate), and at 6 weeks and 6 months. Documentation of current prescribed medications will be obtained from patients, electronic hospital records or general practitioners. This will include:

1. Secondary prevention. Prescription of drug therapy for the prevention of cardiovascular events will be documented; drugs listed will include aspirin, clopidogrel, other anti-platelet agents, statins, angiotensin-converting enzyme inhibitor therapy, and beta-blockers.

2. Pharmacological anti-anginal therapy. Prescription of drug therapy (drug, class, dose) for the alleviation of angina pectoris will be documented; this will include beta-blockades, calcium antagonists, nitrates, nicorandil, and ivabradine.

3. Coronary revascularization. The use of percutaneous coronary intervention and coronary artery bypass surgery will be documented at 6 weeks and 6 months.

\section{Long-term outcomes}

Annual hospitalizations for chest pain episodes, acute coronary syndromes, coronary revascularization procedures, cerebrovascular disease and peripheral vascular disease will be recorded from the Information and Statistics Division of NHS Scotland, and deaths from the Central Registry Office, Scotland for up to 10 years following trial enrolment. Where possible, events will be corroborated by electronic hospital records and case note review.

\section{Diagnosis and management documentation}

The CT scan report will include a section requesting the documentation of any changes in the diagnosis, investigation and treatment of the participant. The chest pain clinic nurse or responsible consultant will complete this pro forma and return it to the trial manager.

At 6 months, trial and data manager will document changes in patient diagnosis, investigation and treatment for all patients at 6 weeks and 6 months using the 


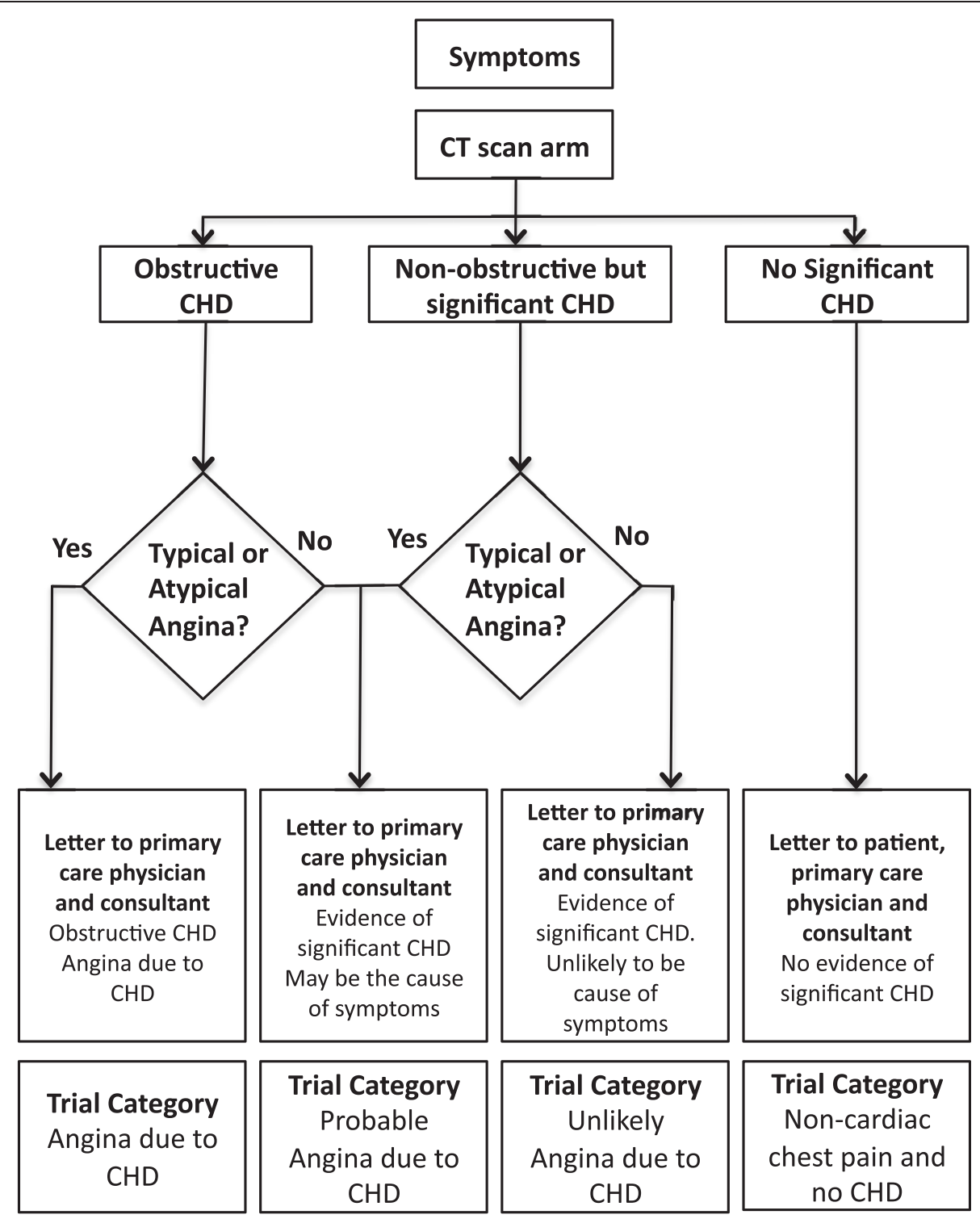

Figure 2 Computed tomography coronary angiography results and diagnosis documentation. (CHD, coronary heart disease; $C T$, computed tomography).

TrakCare $^{\mathrm{TM}}$ software application (InterSystems Corporation, Cambridge, MA, USA), which is an electronic patient record system used by the National Health Service (NHS) Lothian Health Board. This system is destined to be adopted by other centres throughout Scotland. Where appropriate, this will be supplemented by source document review.

\section{Safety outcomes \\ Radiation dose}

The main safety concerns relate to exposure to ionizing radiation [25]. The dose-length product (DLP) will be recorded and the effective radiation dose will be calculated using the conversion factor method. Age- and sex-specific lifetime attributable risks of cancer will be estimated using the Biological Effects of Ionizing Radiation VII Phase 2 report [26]. All incident cancers identified during the study will be recorded throughout the follow-up phase of the trial.

\section{Incidental findings}

Incidental findings occur in 22 to $74 \%$ of CT scans of the chest but only $1.7 \%$ of these are clinically significant [27]. Nevertheless, any incidental findings may require further investigations, involving exposure to ionizing radiation. An incidental finding will be defined as an abnormality identified on $\mathrm{CT}$ without antecedent clinical 


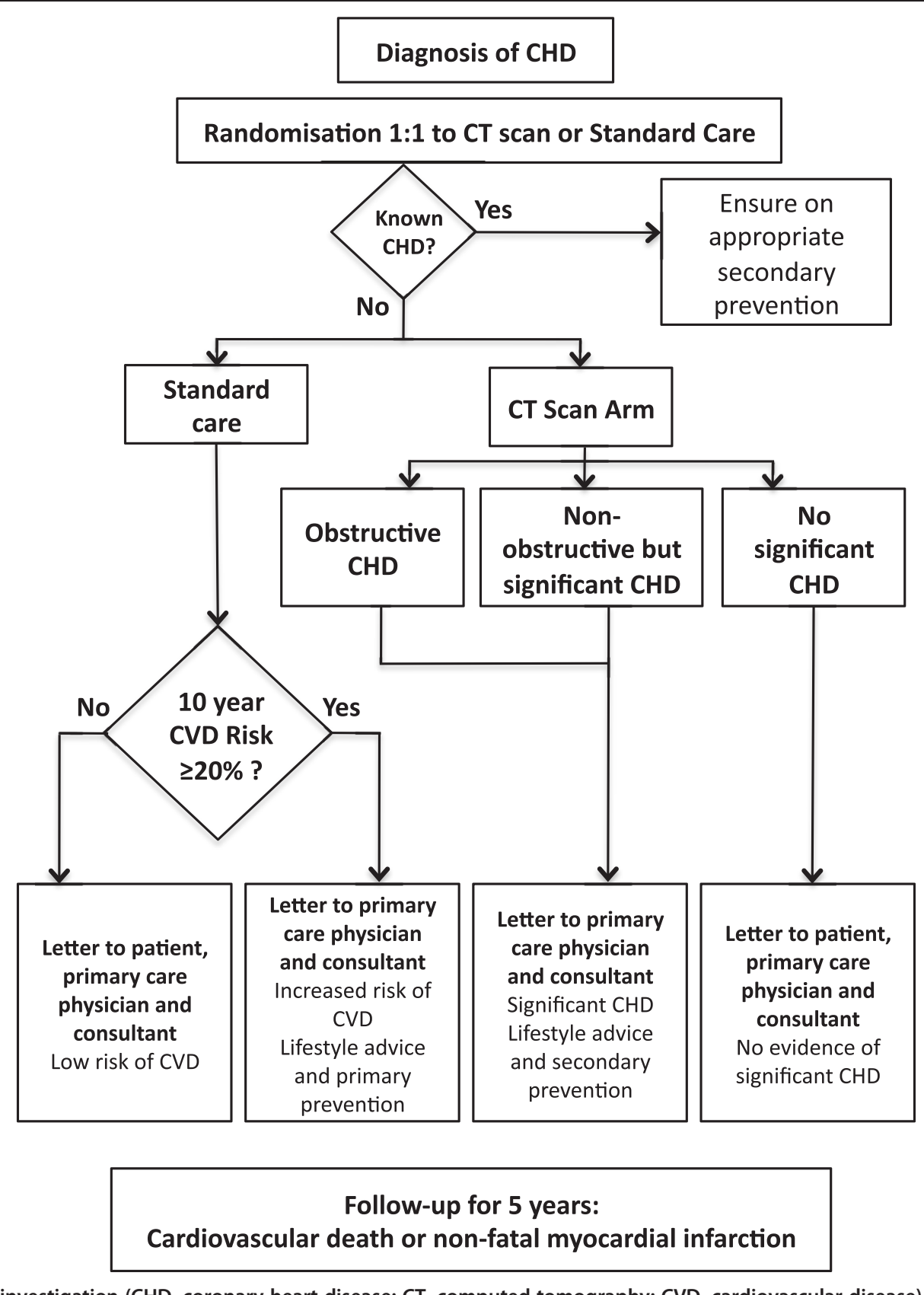

Figure 3 Plan of investigation (CHD, coronary heart disease; $\mathrm{CT}$, computed tomography; CVD, cardiovascular disease).

suspicion or previously known disease [27]. The presence of incidental findings will be documented at the time of the initial CT coronary angiography and any further investigations be documented by review of the participant's medical records.

\section{Data analysis}

The trial results will be reported in accordance with the CONSORT guidelines and, where possible, the clinical profile of unrecruited and ineligible patients will be recorded.

\section{Statistical analysis}

The trial statistician will supervise statistical analyses performed by Edinburgh Clinical Trials Unit. A full statistical analysis plan will be written separately.

\section{Sample size}

Previous studies have diagnosed angina pectoris in $27.0 \%$ of clinic attendees [6]. Whilst CT coronary angiography may reduce 'false-positive' diagnoses of angina; this intervention is most likely to increase the diagnosis of angina, given that current standard diagnostic 
approaches tend to be conservative and under-diagnose coronary heart disease [6]. We believe that, to be clinically useful, this intervention should increase the clinical diagnosis of angina due to coronary heart disease in at least 1 in 25 clinic attendees. For $80 \%$ power at a twosided $P$ value of 0.05 , we will need to recruit 2,069 patients per group to detect an absolute increase of $4.0 \%$ in the diagnosis of angina.

\section{Sample size: long-term outcome}

Establishing the diagnosis of angina is an important aspect for the trial but ultimately it is the long-term patient outcome that is important; can patients' chest pain symptoms be resolved and their long-term outcome improved? After 5 years of follow-up, we would anticipate a coronary event rate (coronary heart disease death or acute coronary syndrome) of $13.1 \%$ for the total population [6]. The study would have $80 \%$ power at a two-sided $P$ value of 0.05 to detect a decrease of $2.8 \%$ in the 5-year event rate. This would also provide $\sim 600$ events and permit the exploration of up to 60 variables [28] in evaluating the predictive value of established risk factors and the novel risk factors of coronary artery calcium score and CT coronary angiography.

\section{Statistical analysis plan}

Where appropriate, two main comparisons will be made: (a) between the scanned and unscanned groups; and (b) in the scanned group only, between the initial clinic assessment and the final assessment following knowledge of the CT scan. The primary analysis will be a comparison between the scanned and unscanned groups, of the proportion of patients diagnosed with angina pectoris secondary to coronary heart disease at 6 weeks. This comparison will be performed using logistic regression, adjusted for the variables in the minimization algorithm.

\section{Health economic analysis}

Health service costs will be assigned to the type and intensity of resource use, measured by the number of diagnostic and therapeutic procedures or interventions, medications, hospital clinic attendances and hospitalization episodes from randomization to 6 months of follow-up. Costs will be attributed to the need for (i) additional invasive or noninvasive imaging, (ii) drug therapy, (iii) coronary revascularization, and (iv) hospitalization for chest pain.

Unit costs will reflect a mixture of approaches, including activity-based analyses of resource consumption for specific procedures or interventions alongside average per diem in-patient costs calculated on a specialtyspecific basis using the Scottish Health Service Costs system. Centre-specific costs for imaging and revascularization procedures/interventions will be determined on the basis of measured procedure duration and the unit costs of these resources for cardiac catheterization laboratories and theatres. Local unit costs for labour, consumables, overheads and depreciation will be obtained from the finance department in each centre. The costs of novel cardiac imaging modalities will be determined using standard 'bottom-up' cost-accounting methods. Costs of hospital admission and out-patient visits will be measured using a 'top-down' costing method. These costs will be estimated for each patient in the trial using centre and specialty-specific average costs, which will also be applied to subsequent in-patient episodes and out-patient attendances beyond 6 months.

The SF- $12{ }^{\circledR}$ and SAQ-UK will be administered by selfcompleted postal questionnaire at baseline and at 6 weeks and 6 months of follow-up. Standard scoring algorithms will be used to calculate the SF- $12^{\circledR}$ health domain profile scales and physical and mental health summary measures (PCS-12 and MCS-12, respectively). The Short Form 6D (SF-6D), a single index preferencebased measure, will be calculated from the SF-12 responses using the Brazier algorithm. Missing hospital cost and quality of life data will be analyzed using multiple imputation techniques.

Cost-effectiveness will be estimated using a prospective within-trial analysis of treatment effects analyzed on an intention-to-treat basis and a decision model of long-term costs and health outcomes. Analysis will be performed from the perspective of the health care system for resource use and the individual patient for health outcomes. The primary endpoint for the economic analysis will be incremental cost-effectiveness ratios comparing the alternative diagnostic strategies' impact on health service use and health related quality of life. The cost-effectiveness analysis will be reported in terms of the incremental cost per quality-adjusted life year (QALY) gained.

The SF-6D utility scores will be combined with survival times to enable estimation of QALYs for all randomized patients. These will be estimated within-trial and over the patients' lifetime, by taking the sum of life years obtained in each arm of the trial within the 6-month follow-up period, modelling subsequent life expectancy and then adjusting expected life-times for observed and modelled SF-6D trajectories.

Information pertaining to resource use, cost, outcome and cost-effectiveness will be reported as the mean per patient in each arm of the trial and the mean difference, with appropriate measures of variance. Cost-effectiveness in sub-groups will be estimated by applying any reduction in overall relative risk or cost to different baseline absolute risk groups. Cost-effectiveness acceptability curves and net benefit statistics will also be reported.

Within-trial analyses will be integrated into a decision model of long-term costs and health effects. The general methods used will follow those defined as good practice 
by the National Institute for Health and Clinical Excellence (NICE). We propose to use a Monte-Carlo microsimulation model, similar to that recently used to assess the cost-effectiveness of 64-MDCT coronary angiography based triage for patients with low-risk chest pain [29]. Base case analysis, using the intention-to-treat results, and sensitivity analysis will be conducted by varying key model parameters and critical assumptions over plausible ranges and distributions. The decision model will also permit the analysis of cost-effectiveness, conditional on pre-specified coronary artery disease risk strata [30] or other important patient characteristics.

\section{Study monitoring}

The trial steering committee and trial management group include representatives from the grant applicants and trial management, as well as individuals not directly involved in the trial. The study is assessing a diagnostic intervention that is unlikely to lead to a major or safetyevent threshold before the end of trial recruitment. Therefore, a data and safety monitoring committee will not be convened.

\section{Discussion}

The multicentre randomized controlled SCOT-HEART trial will assess the added value of CT imaging in over 4,000 patients attending rapid access chest pain clinics. This will define the most appropriate use of this emerging technology in the setting of diagnosing and treating patients with coronary heart disease and angina pectoris. This study will also lay the foundation for future studies to look at the potential prognostic value of this technology.

Current opinion suggests that the main utility of CT coronary angiography lies with the diagnosis and management of patients at intermediate risk of coronary heart disease $[12,20]$ although recent evidence suggests that it may have a role in the care of patients with suspected acute coronary syndrome [31]. However, it could have a role even in apparently high-risk patients with stable symptoms, since CT may help identify patients with 'false-positive' stress tests that could potentially avoid invasive coronary angiography. Alternatively, it may allow early identification of patients at high risk of invasive coronary angiography (such as critical left main stem stenosis), those with anomalous coronary anatomy, or those for whom coronary revascularization is likely to be necessary. In the latter scenario, this will facilitate planning of invasive angiography and allow an interventional cardiologist to undertake follow-on percutaneous coronary intervention as required. This would potentially avoid the need for recurrent invasive angiography in situations where a noninterventional cardiologist performs the diagnostic angiogram.
For apparently low-risk patients, the use of CT coronary angiography is controversial because of the high radiation doses and low pre-test likelihood of disease. This is a particular issue for younger patients and women [25]. However, modern scanners have dramatically reduced radiation exposure, whether because of the increased speed of multidetector scanners that capture the information in a single rotation or through the use of pulse sequences with prospective electrocardiographic gating. This may lead to better focused utilization of secondary preventative therapies in individuals who would otherwise not receive treatment. There is also the added value of imaging noncardiac structures that might be the origin of the presenting chest pain. Finally, this procedure is likely to provide more firm reassurance and potentially more rapid resolution of symptoms in patients anxious to establish whether they have significant coronary heart disease.

Economic evaluation will assist policy makers in deciding whether there is a cost-effective benefit associated with MDCT scans. This is an expensive technology and its healthcare value needs to be established. Potential benefits of MDCT lie in (i) reducing further noninvasive and invasive investigations, (ii) reducing symptoms and improving quality of life through more focused therapy, and (iii) improving long-term clinical outcomes. Thus, measurement of cost-effectiveness requires estimation of resource utilization, quality of life for all patients, and in the subsequent follow-up study, event-free survival.

Without a comprehensive assessment of all patients attending a rapid access chest pain clinic, the utility of $\mathrm{CT}$ will remain undefined and open to question. We believe that this requires a randomized controlled trial to evaluate this emerging and promising imaging technology in a comprehensive and pragmatic manner.

\section{Trial status}

The study has been approved by the South East Scotland Research Ethics Committee. Recruitment is underway at eight sites. At present, 1000 participants have been recruited to the SCOT-HEART study.

\section{Abbreviations \\ AU: Agatston units; CT: Computed tomography; HDL: High-density lipoprotein; MDCT: Multidetector computed tomography; NICE: National institute of clinical excellence.}

\section{Competing interests}

The authors declare that they have no competing interests.

\section{Authors' contribution}

DEN conceived the study, participated in its design and coordination and helped to draft the manuscript. MCW helped to draft the manuscript. All authors read and approved the final manuscript.

\section{Acknowledgements}

DEN (CH/09/002) and MCW (FS/11/014) are supported by the British Heart Foundation. The Clinical Research Imaging Centre (Edinburgh) is supported 
by National Health Service Research Scotland (NRS) through National Health Service Lothian Health Board. The Clinical Research Facility Glasgow and Clinical Research Facility Tayside are supported by National Health Service Research Scotland (NRS).

\section{Funders}

Chief Scientist Office, Scottish Government (CZH/4/588), UK

Edinburgh and Lothian Health Foundation Trust.

Heart Diseases Research Trust.

\section{Sponsor}

The University of Edinburgh and NHS Lothian Health Board act as co-sponsors.

\section{SCOT-HEART Trialists}

Chief Investigator: Professor David Newby.

Trial Research Fellow: Dr Michelle C Williams.

Grant Applicants: Professor David E Newby, Dr Andrew D Flapan, Dr John F

Forbes, Dr Allister D Hargreaves, Professor Stephen J Leslie, Dr Steff C Lewis, Dr Graham McKillop, Dr Scott McLean, Dr John H Reid, Dr James C Spratt,

Dr Neal G Uren.

Trial Steering Committee: Professor Edwin J van Beek, Dr Nicholas A Boon, Mrs Liz Clark, Dr Peter Craig, Dr Marcus D Flather, Dr John F Forbes, Dr Steff C Lewis, Dr Chiara McCormack, Dr Scott McLean, Professor David E Newby, Dr Giles Roditi, Professor Adam D Timmis (Chair), Dr Michelle C Williams. Edinburgh Clinical Trials Unit: Miss Ashma Krishan, Dr Steff C Lewis, Dr Chiara McCormack.

Sites: Borders General Hospital, Melrose: Mrs Gillian Donaldson, Mrs Marlene Fotheringham, Mrs Fiona J Hall, Dr Paul Neary. Forth Valley Royal Hospital, Larbert: Mrs Louisa Cram; Dr Allister D Hargreaves, Ms Sarah Perkins, Dr James C Spratt, Ms Fiona Taylor. Glasgow Royal Infirmary: Dr Hany Eteiba, Dr Alan P Rae, Ms Kate Robb. Ninewells Hospital, Dundee: Ms Dawn Barrie, Ms Kim Bissett, Dr Adelle Dawson, Mr Scot Dundas, Dr Yvonne Fogarty, Dr Prasad Guntur Ramkumar, Professor Graeme J Houston, Mrs Deborah Letham, Ms Linda O'Neill, Professor Stuart D Pringle, Mrs Valerie Ritchie, Dr Thiru Sudarshan, Dr Jonathan Weir-McCall. Royal Alexandra Hospital, Paisley: Dr Alistair Cormack, Dr lain N Findlay, Dr Stuart Hood, Dr Clare Murphy, Dr Eileen Peat. Royal Infirmary of Edinburgh: Ms Barbara Allen, Dr Andrew Baird, Professor Edwin J van Beek, Miss Danielle Bertram, Mr David Brian, Ms Amy Cowan, Dr Nicholas L Cruden, Dr Marc R Dweck, Ms Laura Flint, Miss Samantha Fyfe, Mrs Collette Keanie, Dr Tom J MacGillivray, Dr David S Maclachlan, Miss Margaret MacLeod, Dr Saeed Mirsadraee, Mrs Avril Morrison, Dr Nicholas L Mills, Dr Fiona C Minns, Mrs Alyson Phillips, Miss Laura J Queripel, Dr John H Reid, Dr Nicholas W Weir. St John's Hospital, Livingston; Mrs Fiona Bett, Mrs Frances Divers, Ms Katie Fairley, Dr Ashok J Jacob, Ms Edith Keegan, Ms Tricia White. University Hospital, Ayr: Dr John Gemmill, Mrs Margo Henry, Dr James McGowan. Victoria Hospital, Kirkcaldy: Ms Lorraine Dinnel, Dr C Mark Francis, Mr Dennis Sandeman, Dr Ajay Yerramasu. Western General Hospital, Edinburgh: Mrs Avril Morrison. Western Infirmary, Glasgow/Institute of Cardiovascular \& Medical Sciences, University of Glasgow: Professor Colin Berry, Mrs Heather Boylan, Mrs Ammani Brown, Ms Karen Duffy, Mrs Alison Frood, Mrs Janet Johnstone, Mrs Kirsten Lanaghan, Dr Ross MacDuff, Mr Martin MacLeod, Mrs Deborah McGlynn, Dr Nigel McMillan, Ms Laura Murdoch, Dr Colin Noble, Miss Victoria Paterson, Dr Giles Roditi, Mrs Tracey Steedman, Dr Nikolaos Tzemos.

\section{Author details}

'University of Edinburgh/BHF Centre for Cardiovascular Science, Chancellor's Building, 49 Little France Crescent, Edinburgh EH16 SU4, UK. ${ }^{2}$ Royal Infirmary of Edinburgh, 51 Little France Crescent, Edinburgh EH16 4SA, UK. ${ }^{3}$ School of Economics, University of Edinburgh, 31 Buccleuch Place, Edinburgh EH8 9JT, UK. ${ }^{4}$ Forth Valley Royal Hospital, Larbert FK5 4WR, UK. ${ }^{5}$ Raigmore Hospital, Inverness IV2 3UJ, UK. ${ }^{6}$ Edinburgh Clinical Trials Unit, University of Edinburgh, Western General Hospital, Crewe Road South, Edinburgh EH4 2XU, UK. ${ }^{7}$ Barts Health NHS Trust, The Royal London Hospital, London E1 1BB, UK. ${ }^{8}$ Borders General Hospital, Melrose TD6 9BS, UK. ${ }^{9}$ Clinical Research Imaging Centre, University of Edinburgh, Queen's Medical Research Institute, 47 Little France Crescent, Edinburgh EH16 4TJ, UK. ${ }^{10}$ Peninsula Heart \& Stroke Network, Plymouth PL6 5QZ, UK. ${ }^{11}$ Chief Scientist Office, Scottish Government Health Directorates, St Andrew's House, EdinburghEH1 3DG, UK. ${ }^{12}$ Royal Brompton Hospital, Sydney Street, London SW3 6NP, UK. ${ }^{13}$ Glasgow Royal Infirmary, 16 Alexandra Parade, Glasgow G31 2ER, UK. ${ }^{14}$ Western Infirmary, Glasgow/
Institute of Cardiovascular \& Medical Sciences, University of Glasgow, Glasgow G12 8QQ, UK. ${ }^{15}$ London Chest Hospital, Bonner Road, London E2 9JX, UK. ${ }^{16}$ Ninewells Hospital, Dundee DD1 9SY, UK. ${ }^{17}$ Royal Alexandra Hospital, Paisley PA2 9PN, UK. ${ }^{18}$ Western General Hospital, Edinburgh EH4 2XU, UK. ${ }^{19}$ St John's Hospital, Livingston EH54 6PP, UK. ${ }^{20}$ University Hospital, Ayr KA8 ORX, UK. ${ }^{21}$ Victoria Hospital, Kirkcaldy KY2 5RA, UK.

Received: 2 May 2012 Accepted: 21 September 2012 Published: 4 October 2012

\section{References}

1. Emerson PA, Russell NJ, Wyatt J, Crichton N, Pantin CFA, Morgan AD, Fleming PR: An audit of doctor's management of patients with chest pain in the accident and emergency department. Q J Med 1989, 70:213-220.

2. Tachakra SS, Pawsey S, Beckett M, Potts D: Outcome of patients with chest pain discharged from an accident and emergency department. BMJ 1991, 302:504-505.

3. Gandhi MM, Lampe FC, Wood DA: Incidence, clinical characteristics, and short-term prognosis of angina pectoris. Br Heart J 1995, 79:193-198.

4. Kravitz RL, Laouri M, Kahan JP, Guzy P, Sherman T, Hilborne L, Brook RH: Validity of criteria used for detecting underuse of coronary revascularization. JAMA 2012, 274:632-638.

5. Newby DE, Fox KA, Flint LL, Boon NA: A "same day" direct-access chest pain clinic: improved management and reduced hospitalization. Q J Med 1998, 91:333-337.

6. Sekhri $N$, Feder GS, Junghans $C$, Hemingway $H$, Timmis AD: How effective are rapid access chest pain clinics? Prognosis of incident angina and non-cardiac chest pain in 8762 consecutive patients. Heart 2007, 93:458-463.

7. Boyle RM: Value of rapid-access chest pain clinics. Heart 2007, 93:415-416.

8. Pletcher MJ, Tice JA, Michael P, Browner WS: Using the coronary artery calcium score to predict coronary heart disease events: a systematic review and meta-analysis. Arch Internal Medicine 2004, 164:1285-1292.

9. Raggi P, Cooil B, Shaw L, Aboulhson J, Takasu J, Budoff M, Callister TQ: Progression of coronary calcium on serial electron beam tomographic scanning is greater in patients with future myocardial infarction. Am J Cardiol 2003, 92:827-829.

10. Nicholls SJ, Tuzcu EM, Wolski K, Sipahi I, Schoenhagen P, Crowe T, Kapadia SR, Hazen SL, Nissen SE: Coronary artery calcification and changes in atheroma burden in response to established medical therapies. JACC 2007, 49:263-270.

11. Houslay ES, Cowell SJ, Prescott RJ, Reid J, Burton J, Northridge DB, Boon NA Newby DE: Progressive coronary calcification despite intensive lipid-lowering treatment: a randomised controlled trial. Heart 2006, 92:1207-1212

12. Rubinshtein R, Halon DA, Gaspar T, Jaffe R, Karkabi B, Flugelman MY, Kogan A, Shapira R, Peled N, Lewis BS: Usefulness of 64-slice cardiac computed tomographic angiography for diagnosing acute coronary syndromes and predicting clinical outcome in emergency department patients with chest pain of uncertain origin. Circulation 2007, 115:1762-1768.

13. Kopp A, Schroeder S, Kuettner A, Baumbach A, Georg C, Kuzo R, Heuschmid M, Ohnesorge B, Karsch K, Claussen C: Non-invasive coronary angiography with high resolution multidetector-row computed tomography. Results in 102 patients. Eur Heart J 2002, 23:1714-1725.

14. Houslay ES, Lawton T, Sengupta A, Uren NG, McKillop G, Newby DE: Non-invasive assessment of coronary artery bypass graft patency using 16-slice computed tomography angiography. J Cardiothorac Surg 2007, 2:27.

15. Leber AW, Becker A, Knez A, von Ziegler F, Sirol M, Nikolaou K Ohnesorge B, Fayad ZA, Becker CR, Reiser M, Steinbeck G, Boekstegers P: Accuracy of 64-slice computed tomography to classify and quantify plaque volumes in the proximal coronary system: a comparative study using intravascular ultrasound. JACC 2006, 47:672-677.

16. Schroeder S, Achenbach S, Bengel F, Burgstahler C, Cademartiri F, de Feyter P, George R, Kaufmann P, Kopp AF, Knuuti J, Ropers D, Schuijf J, Tops LF, Bax JJ: Cardiac computed tomography: indications, applications, limitations, and training requirements. Eur Heart J 2008, 29:531-536.

17. Achenbach S: Computed tomography coronary angiography. JACC 2006, 48:1919-1928.

18. Mowatt G, Cummins E, Waugh N, Walker S, Cook J, Jia X, Hillis GS, Fraser C Systematic review of the clinical effectiveness and cost-effectiveness of 
64-slice of higher computed tomography angiography as an alternative to invasive coronary angiography in the investigation of coronary artery disease. Health Technol Assess 2008, 12:1386-1393.

19. National Institute for Health and Clinical Excellence: Chest Pain of Recent Onset: Assessment and Diagnosis of Recent Onset Chest Pain or Discomfort of Suspected Cardiac Origin. 95th edition. London: NICE Clinical Guideline; 2010.

20. National Institute for Health and Clinical Excellence: Chest Pain of Recent Onset: Assessment and Investigation of Recent Onset Chest Pain or Discomfort of Suspected Cardiac Origin. Full Guideline - Consultation Version. London: 2009.

21. Agatston AS, Janowithz WR, Hildner FJ, Zusmer NR, Viamonte M, Detrano R: Quantification of coronary artery calcium using ultrafast computed tomography. JACC 1990, 15:827-827.

22. McClelland RL, Chung H, Detrano R, Post W, Kronmal RA: Distribution of coronary artery calcium by race, gender, and age: results from the Multi-Ethnic Study of Atherosclerosis (MESA). Circulation 2006, 113:30-37.

23. Austen WG, Edwards JE: A reporting system on patients evaluated for coronary artery disease. Report of the Ad Hoc Committee for Grading of Coronary Artery Disease, Council on Cardiovascular Surgery, American Heart Association. Circulation 1975, 51:5-40.

24. Spertus JA, Winder JA, Dewhurst TA, Deyo RA, Prodzinski J, McDonell M, Fihn SD: Development and evaluation of the Seattle Angina Questionnaire: a new functional status measure for coronary artery disease. JACC 1995, 25:333-341.

25. Einstein AJ, Henzlova MJ, Rajagopalan S: Estimating risk of cancer associated with radiation exposure from 64-slice computed tomography coronary angiography. JAMA 2007, 298:317-323.

26. Committee to Assess Health Risks from Exposure to Low Levels of lonizing Radiation: Nuclear and Radiation Studies Board, Division on Earth and Life Studies NRC of the NA. Health Risks from Exposure to Low Levels of lonizing Radiation: BEIR VII Phase 2. Washington, DC: National Academies Press; 2006.

27. Machaalany J, Yam Y, Ruddy TD, Abraham A, Chen L, Beanlands RS, Chow BJW: Potential clinical and economic consequences of noncardiac incidental findings on cardiac computed tomography. J Am Coll Cardiol 2009, 54:1533-1541.

28. Harrell FE, Lee KL, Mark DB: Multivariable prognostic models: issues in developing models, evaluating assumptions and adequacy, and measuring and reducing errors. Stat Med 1996, 15:361-387.

29. Ladapo JA, Hoffmann U, Bamberg F, Nagurney JT, Cutler DM, Weinstein MC, Gazelle GS: Cost-effectiveness of coronary MDCT in the triage of patients with acute chest pain. Am J Roentgenol 2008, 191:455-463.

30. Kreisz FP, Merlin T, Moss J, Atherton J, Hiller JE, Gericke CA: The pre-test risk stratified cost-effectiveness of 64-slice computed tomography coronary angiography in the detection of significant obstructive coronary artery disease in patients otherwise referred to invasive coronary angiography. Heart Lung Circ 2009, 18:200-207.

31. Investigators ROMICAT-II, Hoffmann U, Truong QA, Schoenfeld DA, Chou ET, Woodard PK, Nagurney JT, Pope JH, Hauser TH, White CS, Weiner SG, Kalanjian S, Mullins ME, Mikati I, Peacock WF, Zakroysky P, Hayden D, Goehler A, Lee H, Gazelle GS, Wiviott SD, Fleg JL, Udelson JE: Coronary CT angiography versus standard evaluation in acute chest pain. $N$ Engl J Med 2012, 367:299-308.

doi:10.1186/1745-6215-13-184

Cite this article as: Newby et al:: Role of multidetector computed tomography in the diagnosis and management of patients attending the rapid access chest pain clinic, The Scottish computed tomography of the heart (SCOT-HEART) trial: study protocol for randomized controlled trial. Trials 2012 13:184

\section{Submit your next manuscript to BioMed Central and take full advantage of:}

- Convenient online submission

- Thorough peer review

- No space constraints or color figure charges

- Immediate publication on acceptance

- Inclusion in PubMed, CAS, Scopus and Google Scholar

- Research which is freely available for redistribution

Submit your manuscript at www.biomedcentral.com/submit 